\section{Language habits, acoustic confusability, and immediate memory for redundant letter sequences*}

\author{
A. D. BADDELEY \\ Experimental Psychology Laboratory, University of Sussex \\ Brighton BN1 9QY, Sussex, England
}

Immediate memory was tested for sequences of $7,8,9$, or 10 auditorily presented letters which comprised either words or zero-, first-, second-, or third-order approximations to English words. At all lengths, recall probability correlated highly with letter sequence predictability (.58-.78) but was unrelated to acoustic confusability. It is suggested that coding was still phonemic but involved speech sounds comprising several letters rather than letter names.

Short-term memory for letter sequences is influenced by two major variables, predictability and acoustic confusability. Predictability is a measure of the closeness with which a letter sequence approximates to the structure of English, and has been shown to correlate highly with probability of recalling approximations to English words (Baddeley, 1964a), and somewhat less highly with the recall of sequentially presented consonant sequences (Baddeley, Conrad, \& Hull, 1965). A series of studies have shown a high correlation between probability of recalling a visually presented consonant sequence and its acoustic confusability, as measured by the probability of mishearing the constituent letters when presented in noise (Conrad, 1962; Conrad \& Hull, 1964). A later study by Conrad, Freeman, \& Hull (1965) directly compared the contributions of predictability and acoustic confusability to the recall of sequences of six random consonants and concluded that the main determinant of recall probability in this situation was acoustic confusability, with predictability playing a relatively minor role. Kintsch (1970, p. 191) has subsequently concluded, on the basis of this one very limited study, that "Language factors appear to be ineffective in short-term memory, or at least are overridden in importance by acoustic confusions."

Such a conclusion is clearly at variance with the results of Baddeley (1964a). However, direct comparison is difficult,

*This study was performed while the author was at the Medical Research Council Applied Psychology Unit Cambridge, and the report written while visiting the Center for Human Information Processing, Lniversity of California, San Diego. I am grateful to Mrs. P. Altham and Mrs. A. J. Hull for assistance with data analysis and to the Medical Research Council and to the National Institute of Mental Health. Grant WH 15828 , for financial support. since Baddeley's study did not include measures of acoustic confusability. Furthermore, all the letters in each sequence were presented simultaneously, a procedure that recent work suggests may minimize acoustic confusability effects (Adams, Thorsheim, \& McIntire, 1969; Laverty \& Turvey, 1970). The following experiment, therefore, uses the technique that seems most likely to favor acoustic coding, sequential auditory presentation. and studies the relationship between acoustic confusability, predictability, and probability of recall of letter sequences of varying approximation to English.

\section{MATERIAL}

The technique described by Miller, Bruner, \& Postman (1954) was used to generate eight sequences of $7,8,9$, and 10 letters of zero-, first-, second-, and third-order approximation to English. These were based on the tables of letter frequency in written Engish compiled by Baddeley, Conrad, \& Thomson (1960). In addition, four sets of eight words (one set each of length $7,8,9$, and 10 letters) were selected at random from the Thorndike-Lorge list, making a total of 40 sequences of each length.

\section{PROCEDURE}

A separate group of 20 enlisted men was tested at each of the four sequence lengths. Letters were read out at a rate of one per second, and Ss were allowed $2 \mathrm{sec}$ per letter to recall as much of the sequence as possible. Ss were instructed to write each letter in the appropriate serial position on a prepared answer sheet. They were familiarized with the material to be used sequence of each of the five approximations, which were read out at a rate of one letter per second. Group testing was used. The 40 sequences at each length were presented in random order.

RESULTS

Recall was scored in terms of number of by being required to copy out one letters reprodaced in the appropriate seriul position. Figure I shows mean number of crrors as a function of redundancy and sequence length. The redundancy of each approximation was computed on the basis of Shannon's $(1951)$ estimate of the redundancy of English. Lorrected for sequence length (Baddeley, 1964b). For al lengths of sequence, there is a linear relationship between error rate and redundancy. This trend is shown by virtually all of the 80 Si lested and indicates that the affect of letter-sequence redundancy is not dependent on simultaneous visual presentation.

Shannon's redundancy estimates were used to compute the mean amount of information reproduced in the varying conditions, ignoring any information contained in errors, and is shown in Fig. ?. The optimal order of approximation from the viewpoint of conveying information changes with sequence length from zero order at 7. to first order at 8 . second order at 9 , and third order at 10 . Overall. however, the maximum information is conveyed in the zero-order sequences. though this is probably due largely to a ceiling effect caused by the very limited informational capacity of the shorter redundant sequences. Nevertheless, it appears to be the case that, although redundancy increases number of .items recalled, at no point does it substantially increase amount of information reproduced. This is consistent with the results of Pollack (1953), who manipulated information content by varying the basic vocabulary size.

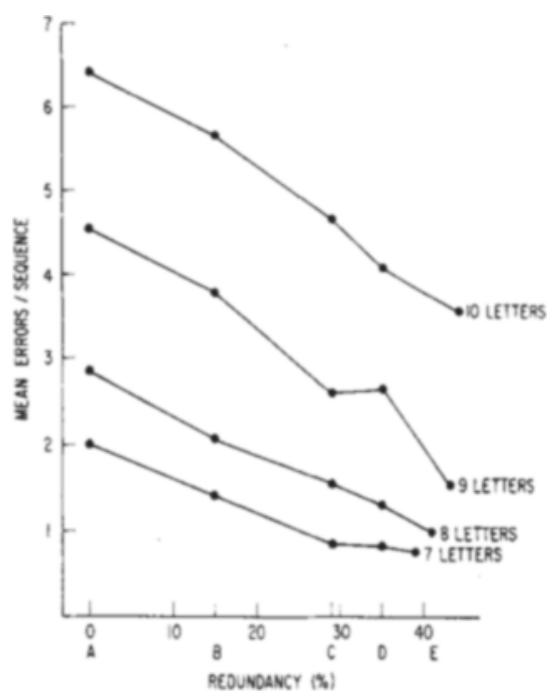

Fig. 1. Mean number of errors per sequence as a function of length and redundancy. $A=$ zero order, $B=$ first order, $C=$ second order, $D=$ third order, $E$ $=$ words. 


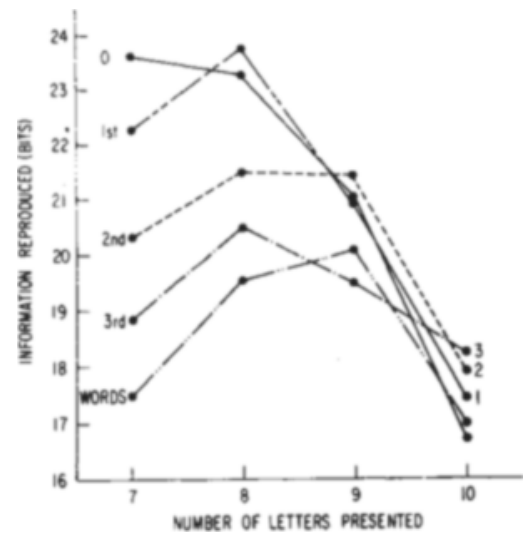

Fig. 2. Mean amount of information reproduced (bits) as a function of sequence length and order of approximation to English.

Mean percentage of letters recalled was then computed for each sequence and was correlated with acoustic confusability (Conrad \& Hull, 1964) and predictability (Baddeley, 1964a) using the product-moment correlation. Results are shown in Table 1. It is clear that performance is highly correlated with predictability at all sequence lengths. Acoustic confusability of the sequences, on the other hand, shows no overall relationship to recall. A significant correlation occurs for sequences of length 10 but is in the direction of better performance on confusable sequences. This is probably due to a positive correlation between confusability and predictability ( $r=.429, p<.01)$ for this length of sequence, which, in turn, probably reflects the high frequency of confusable Ts and Es and of repetitions in long predictable sequences.

\section{DISCUSSION}

This result suggests that, although the sequences were presented as isolated letter names, Ss did not encode them in that way. It seems likely that they combined letters to form composite sounds (e.g., B-E-D will be recoded as the word "bed" rather than be stored as three independent letters). Under such conditions, the letter names become irrelevant. Predictable sequences that conform to S's language habits will be encoded more easily and into fewer chunks, and are hence more likely to be recalled (Miller, 1956). While such coding will be simplest with predictable material, recent failures to observe acoustic similarity effects (Adams, Thorsheim, \& McIntire, 1969; Laverty \& Turvey, 1970) suggest that it may also occur with consonant sequences, given appropriate conditions. Such results certainly indicate that Ss are not coding in terms of letter names; they do not, however, mean that

Table 1

Correlation with Mean Recall Score of Letter Sequence Predictability and Acoustic Confusability for Sequences of 7 to 10 Letters

\begin{tabular}{|c|c|c|c|c|c|}
\hline & 7 & 8 & 9 & 10 & Overall \\
\hline Predictability & $.578^{* *}$ & $.743^{* *}$ & $.782 * *$ & $.750 * *$ & $.655^{* *}$ \\
\hline Acoustic Confusability & .081 & .022 & .184 & $.338 *$ & .050 \\
\hline
\end{tabular}

$* p<.05, *{ }^{*} p<.001$

the coding is nonacoustic, as Adams et al imply.

In conclusion, it appears that language habits may have a marked effect on STM. This is reflected by the correlation between predictability and recall probability, which suggests that $S$ is recoding the letter names presented into speech sounds. It does not imply that the coding is no longer phonemic.

\title{
REFERENCES
}

ADAMS, J. A., THORSHEIM, H. E., \& McINTIRE, J. S. Item length, acoustic similarity and natural language mediation as variables in short-term memory. Journal of Experimental Psychology, 1969, 80, 39-46.

BADDELEY, A. D. Immediate memory and the "perception" of letter sequences. Quarterly Journal of Experimental Psychology, 1964a, 16, 364-367.

BADDELEY, A. D. Space information and the redundancy of letter sequences. American Journal of Psychology, 1964b, 77, 322.

BADDELEY, A. D., CONRAD, R., \& HULL, A J. Predictability and immediate memory for consonant sequences. Quarterly Journal of Experimental Psychology, 1965, 17, 175-177. BADDELEY, A. D., CONRAD, R., \&

\section{THOMSON, W. E. Letter structure of the \\ Vividness in the recall of English nominalizations*}

\author{
ALEXANDER J. WEARING \\ Yale University, New Haven, Conn. 06510
}

Rohrman (1968) presented data which purported to show that variations in deep structure could predict the recall of English nominalizations that had identical surface structures. Evidence is presented which suggests that semantic vividness was confounded with Rohrman's experimental manipulations, and it is argued that his results can be explained solely in terms of vividness.

Conventional wisdom has it that the difficulty of remembering a sentence is determined mainly by its deep structure (Garrett \& Fodor, 1968). A dissenting view comes from Martin \& Roberts (1966), who proposed that recall is predicted better by the surface than by the deep structure of a sentence.

In a most interesting series of experiments, Rohrman (1968) tested the relative merits of these two arguments by comparing word strings that had different deep structures but identical surface

*The author is grateful to Theodore K. Phelps and Rosemary Wearing for assistance in the collection and analysis of the data and to Rosemary Wearing for criticism of an earlier draft of this paper. structures. Consider the subject nominalization growling lions and the object nominalization digging holes. Both are dominated by a NP node and both consist of a participle and a noun. They have identical surface structures. However, their underlying structures are not the same. The subject nominalization is derived from deep structures of the form (lions growl), whereas the object nominalization derives from (PRO(dig holes)), where PRO represents an indefinite nominal functioning as the subject. In addition to the difference in node complexity, the transformational histories of the nominalizations differ in that the object nominalization requires a deletion transformation to reach the surface form, whereas the subject nominalization 